\title{
ON WEAK HARMONIC MAASS - MODULAR GRIDS OF EVEN INTEGRAL WEIGHTS
}

\author{
P. GuerzhoY*
}

\begin{abstract}
A number of examples of families of weak Maass forms and modular forms which satisfy a striking equality between their $q$-expansion coefficients appeared recently. One can formulate this equality by saying that these coefficients constitute a grid. In this paper we consider the simplest setting of even integral weight and full modular group. We prove that for every positive even integral weight a grid exists and is unique.
\end{abstract}

\section{Introduction}

For a $k \in \frac{1}{2} \mathbb{Z}$ denote by $M_{\dot{k}}^{!}(N)$ the space of weakly holomorphic (i.e. those who may have their poles only at the cusps) modular forms of weight $k$ on $\Gamma_{0}(N)$. As usual, $M_{k}(N)$ and $S_{k}(N)$ are the spaces of weight $k$ modular and cusp forms correspondingly on $\Gamma_{0}(N)$. Let $N_{2-k}(N)$ be the space of weight $2-k$ harmonic weak Maass forms on $\Gamma_{0}(N)$ which satisfy the property $\xi(g)=\xi_{2-k}(g) \in S_{k}$ for every $g \in N_{2-k}(N)$. We refer to $[4,5]$ for the definitions relevant to harmonic weak Maass forms and the operator $\xi: N_{2-k} \rightarrow S_{k}$. Note that our $N_{2-k}(N)$ is denoted by $\operatorname{Maass}_{2-k}^{*}\left(\Gamma_{0}(N)\right)$ in [4]. Recall that every $g \in N_{2-k}(N)$ may be uniquely decomposed $g=g^{-}+g^{+}$into a sum of its non-holomorphic and holomorphic components.

Definition 1. We call two collections $f_{n} \in M_{\dot{k}}^{!}(N)$ and $g_{m} \in N_{2-k}(N)$ with qexpansions

$$
\begin{gathered}
f_{n}=q^{-n}+\sum_{m>0} a(m, n) q^{m}, \quad n \geq 0 \\
g_{m}=g_{m}^{-}+q^{-m}+\sum_{n \geq 0} b(m, n) q^{n}, \quad m>0
\end{gathered}
$$

a weak Maass - modular grid (or simply a grid) of weight $k$ on $\Gamma_{0}(N)$ if the identity of Fourier coefficients

$$
a(m, n)=-b(m, n)
$$

holds. The indices $n$ and $m$ must satisfy $n \geq 0$ and $m>0$, and may belong to some arithmetic progressions.

Although the very fact of the existence of grids looks astonishing, many examples were recently found. The first one was discovered by Zagier [12] (with $k=1 / 2, N=$ $4, \quad n \equiv 0,3(4), \quad m \equiv 0,1(4))$. In this case $g_{m} \in M_{3 / 2}^{!} \subset N_{3 / 2}(4)$. Zagier's construction was recently generalized to other half-integral weights $k$ by Bringmann and

Received by the editors March 10, 2008.

1991 Mathematics Subject Classification. 11F11,11F12.

* Supported by NSF grant DMS-0700933. 
Ono [3]. Another example (with $k=3 / 2, \quad N=144, \quad n \equiv-1(24), \quad m \equiv 1(24)$, the modular and harmonic weak Maass forms have non-trivial Nebentypus) was recently found by Folsom and Ono [9]. The latter example is especially interesting for its close connection to the Ramanujan mock theta-function: the Fourier coefficients of $g_{1}^{+}$coincide with those of the Ramanujan mock theta-function. Note that similar identities between Fourier coefficients appear implicitly in earlier works on non-holomorphic Poincaré series $[11,10,8]$.

In this paper we consider the grids of even integral weight $k$ on the full modular group $S L_{2}(\mathbb{Z})$. We thus assume $N=1$ and drop it from our notations.

In the case of even integral weight $k$, grids appear in [1] for small values of $k$, (namely, $k=0,4,6,8,10,14$ ). In these cases the spaces of cusp forms of weight $k$ are empty, and [5, Proposition 3.5] implies that $g_{m} \in M_{2-k}^{!}$. The results of [1] were recently generalized in [7] to arbitrary even $k$. Another type of grids, which are essentially different from ours and do not involve harmonic weak Maass forms appears in [7] in this connection.

There is nothing mysterious about the $k=2$ case which is not mentioned in [1].

Proposition. There exists a unique grid of weight 2 with indexes $n$ running through all non-negative integers and $m$ through all positive integers.

Proof. Notice firstly that $f_{0}$ must vanish since $M_{2}$ is empty. Condition (1) implies that the $g_{m}$ have no constant terms. It follows that $g_{m}$ is a weight zero weakly holomorphic modular form ([5, Proposition 3.5] implies that $g_{m} \in M_{0}^{!}$since $S_{2}$ is empty), which has a principal part of $q^{-m}$ and no constant term. Such a form exists and is unique (this is the $m$-th Faber polynomial in the modular invariant $j$ ). The uniqueness of $g_{m}$ along with condition (1) implies the uniqueness clause. For the existence clause, denote by $D$ the differential operator

$$
D=q \frac{d}{d q},
$$

which takes $S L_{2}(\mathbb{Z})$-invariant functions to modular forms of weight 2. For $n \geq 1$, let $f_{n}=-(1 / n) D g_{n}$. Condition (1) follows now from the fact that the product $f_{n} g_{m}$, for every $n, m \geq 1$, is a weakly holomorphic modular form of weight 2 , therefore, a derivative of a polynomial in $j$, and thus has no constant term.

Our main result is completely similar to that of the above Proposition.

Theorem 1. Let $k \geq 0$ be an even integer.

There exists a unique grid of weight $k$ with indexes $n$ running through all nonnegative integers and $m$ through all positive integers.

Moreover, we have

$$
f_{n}=-n^{1-k} D^{k-1} g_{n}^{+}
$$

Remark 1. If $k=0,4,6,8,10,14$, then our functions $f_{n}$ and $g_{m}$ coincide with those introduced and studied in [1]. We actually prove Theorem 1 under the assumption $k>2$. The case $k=2$ is taken care of in the Proposition above.

Remark 2. Our approach is different from that of [3, 9]: we avoid the explicit calculation of Fourier coefficients of Poincaré series in terms of Bessel functions. In fact, 
we need only one Fourier coefficient of these series, which equals 1, and is relatively easy to calculate.

A recent work of Bruinier and Ono [6, Theorem 1.1(2)] indicates how important the algebraicity of the Fourier coefficients of the holomorphic part of a harmonic weak Maass form may be. In this connection we present the following corollary which states that the Fourier coefficients of all $f_{n}$ for $n>0$ are rational up to, in a sense, one cusp form of weight $k$, and, by (2), the Fourier coefficients of $g_{n}^{+}$are rational up to its Eichler integral.

Corollary 1. For a weight $k$ grid $\left(f_{n}, g_{m}\right)$ there exists a cusp form $\psi \in S_{k}$ such that

$$
f_{n}-n^{1-k} \psi \mid T_{n} \in \mathbb{Q}\left[q^{-1}, q\right],
$$

where $T_{n}$ denotes the usual weight $k$ Hecke operator.

We state some propositions and prove Theorem 1 in Section 2 of the paper. We prove these propositions and Corollary 1 in Section 3.

\section{Proof of Theorem 1}

We begin with the existence and uniqueness of the harmonic weak Maass part of the grid.

Proposition 1. For every positive integer $m$ there exists a unique harmonic weak Maass form $g_{m} \in N_{2-k}$ such that $g_{m}=g_{m}^{-}+g_{m}^{+}$and

$$
g_{m}^{+}=q^{-m}+\mathcal{O}(1) \text {. }
$$

The existence of $g_{m}$ follows immediately from [4, Proposition 3.3]. The uniqueness, which we prove here, shows how natural the construction of fundamental Poincaré series in [4] is.

Remark 3. As it was indicated by the referee, the existence of $g_{m}$ can be also derived from [5, Proposition 3.11]. In this way one uses only basic facts on the cohomology of Riemann surfaces, and avoids the consideration of Poincaré series.

The uniqueness clause of Theorem 1 follows immediately from Proposition 1.

In order to establish the existence clause of Theorem 1, we firstly prove the following generalization of a classical result of Bol [2].

Proposition 2. If $g=g^{-}+g^{+} \in N_{2-k}$, then $D^{k-1} g^{+} \in M_{k}^{!}$.

Note that Bol's identity immediately implies Proposition 2 if $g^{-}=0$ (i.e. $g \in$ $\left.M_{2-k}^{!}\right)$.

In order to formulate the last proposition, let us introduce a bilinear pairing between the spaces $M_{k}^{!}$and $N_{2-k}$. If $f \in M_{k}^{!}$and $g=g^{-}+g^{+} \in N_{2-k}$, put

$$
\{f, g\}=\text { the constant term of the product } f g^{+}
$$

Denote by $(\cdot, \cdot)$ the Petersson inner product. A result of Bruinier and Funke [5, Proposition 3.5] specializes in our setting to the equality

$$
\{f, g\}=(f, \xi(g))
$$


if the right-hand side converges (this is the case every time when we use this fact below, because we calculate the left-hand side by the means of the Petersson product in the right). This allows us to identify $f_{0}$ in our grid as an Eisenstein series

$$
f_{0}:=E_{k}=1-\frac{2 k}{B_{k}} \sum_{m>0} \sigma_{k-1}(m) q^{m},
$$

where, as usual, $B_{k}$ is the $k$-th Bernoulli number, and $\sigma_{k-1}(m)=\sum_{d \mid m} d^{k-1}$. In other words,

$$
a(m, 0)=-b(m, 0)=-\frac{2 k}{B_{k}} \sigma_{k-1}(m) .
$$

Indeed, since $g_{m} \in N_{2-k}$, we have $\xi\left(g_{m}\right) \in S_{k}$, and therefore $\left(f_{0}, \xi\left(g_{m}\right)\right)=0$. It follows that $\left\{f_{0}, g_{m}\right\}=0$ for all $m>0$. That is $a(m, 0)=-b(m, 0)$ as required.

Identity (4) checks well with part 3 of [4, Proposition 3.3], where the constant term of the Poincaré series is calculated.

Since

$$
\left\{f_{n}, g_{m}\right\}=a(m, n)+b(m, n),
$$

Theorem 1 now follows from Propositions 1 and 2 combined with the following statement

Proposition 3. For $n, m>0$,

$$
\left\{D^{k-1} g_{n}^{+}, g_{m}\right\}=0 .
$$

\section{Proofs of Propositions $1,2,3$, and Corollary 1}

Proof of Proposition 1. As it is mentioned above, we only need to prove the uniqueness. This uniqueness follows from the fact that an element of $N_{2-k}$ is defined by its principal part uniquely. Indeed, assuming the existence of two elements with the same principal part we consider their difference, say, $h \in N_{2-k}$, which has no principal part, and obtain $0=\{\varphi, h\}=(\varphi, \xi(h))$ for every $\varphi \in S_{k}$. It follows that $\xi(h)=0$, therefore $h \in M_{2-k}^{!}$. Thus $h=0$ since it has no principal part and $k>0$.

We now calculate $\xi\left(g_{m}\right)$. Let $\varphi_{i}=\sum_{n>1} \lambda_{i}(n) q^{n}$ with $i=1, \ldots, \operatorname{dim} S_{k}$ be the basis of the space of weight $k$ cusp forms $\bar{S}_{k}$ which consists of Hecke eigenforms $\varphi$ normalized by the condition $\lambda_{i}(1)=1$. Since $g_{m} \in N_{2-k}$, there exist $\alpha_{i}(m) \in \mathbb{C}$ such that

$$
\xi\left(g_{m}\right)=\sum_{i=1}^{\operatorname{dim} S_{k}} \alpha_{i}(m) \varphi_{i} .
$$

We combine the above equality with (3) to obtain

$$
\lambda_{i}(m)=\left\{\varphi_{i}, g_{m}\right\}=\left(\varphi_{i}, \xi\left(g_{m}\right)\right)=\alpha_{i}(m)\left(\varphi_{i}, \varphi_{i}\right) .
$$

Since the Petersson scalar product is non-degenerate, $\left(\varphi_{i}, \varphi_{i}\right) \neq 0$, we have

$$
\xi\left(g_{m}\right)=\sum_{i=1}^{\operatorname{dim} S_{k}} \lambda_{i}(m) \frac{\varphi_{i}}{\left(\varphi_{i}, \varphi_{i}\right)} .
$$


Note for further use that we can reformulate (5) as the action of the Hecke operator $T_{m}$ :

$$
\xi\left(g_{m}\right)=\xi\left(g_{1}\right) \mid T_{m}
$$

Recall that for a non-negative integer $l$ the weight $l$ action of a matrix $\gamma=$ $\left(\begin{array}{ll}a & b \\ c & d\end{array}\right) \in S L_{2}(\mathbb{Z})$ on a function $G$ on the upper half-plane $\Im \tau>0$ is defined by

$$
\left(\left.G\right|_{l} \gamma\right)(\tau)=(c \tau+d)^{-l} G\left(\frac{a \tau+b}{c \tau+d}\right)
$$

Bol's identity states that this action intertwines with $l-1$ differentiations, namely for any differentiable function $G$

$$
D^{l-1}\left(\left.G\right|_{2-l} \gamma\right)=\left.\left(D^{l-1} G\right)\right|_{l} \gamma .
$$

Proof of Proposition 2. Let $f=\xi(g) \in S_{k}$, and let

$$
\mathcal{F}_{f}(z)=\int_{-\bar{z}}^{i \infty} \frac{\overline{(f(-\bar{\tau}))}}{(-i(\tau+z))^{2-k}} d \tau \text {. }
$$

It follows from (the proof of) [4, Theorem 1.1] that there exists a holomorphic function on the upper half-plane $g_{h}$ such that the function $\tilde{g}$ defined by

$$
\tilde{g}=g_{h}+A \mathcal{F}_{f}(z)
$$

with a certain $A \in \mathbb{C}^{*}$ is a harmonic weak Maass form, $\tilde{g} \in N_{2-k}$. Moreover, $\xi(\tilde{g})=f$, and $\tilde{g}^{+}=g_{h}$. We apply [5, Proposition 3.2] to conclude that $g^{+}-g_{h} \in M_{2-k}^{!}$. Bol's identity now implies that

$$
M_{k}^{!} \ni D^{k-1}\left(g^{+}-g_{h}\right)=D^{k-1}\left(g^{+}\right)-D^{k-1}\left(g_{h}\right) .
$$

In order to finish the proof we need to show that $D^{k-1}\left(g_{h}\right) \in M_{k}^{!}$. Bol's identity implies that $D^{k-1} \tilde{g}$ is invariant with respect to the weight $k$ action of $S L_{2}(\mathbb{Z})$, Thus for every $\gamma \in S L_{2}(\mathbb{Z})$

$$
\left.\left(D^{k-1} g_{h}\right)\right|_{k} \gamma+\left.A\left(D^{k-1} \mathcal{F}_{f}\right)\right|_{k} \gamma=D^{k-1} g_{h}+A D^{k-1} \mathcal{F}_{f},
$$

and our claim will follow from the invariance of $D^{k-1} \mathcal{F}_{f}$ with respect to the weight $k$ action of $S L_{2}(\mathbb{Z})$. A substitution calculation, which takes into the account that $\overline{(f(-\bar{\tau}))} \in S_{k}$, implies that, for every $\gamma=\left(\begin{array}{ll}a & b \\ c & d\end{array}\right) \in S L_{2}(\mathbb{Z})$,

$$
\mathcal{F}_{f}(z)-\left(\left.\mathcal{F}_{f}\right|_{2-k} \gamma\right)(z)=P(z)
$$

where

$$
P(z)=(i)^{k-2} \int_{d / c}^{i \infty}(z+\tau)^{k-2} \overline{(f(-\bar{\tau}))} d \tau
$$

is a polynomial in $z$ of degree at most $k-2$. Thus

$$
\left.0=D^{k-1}\left(\mathcal{F}_{f}(z)-\left.\mathcal{F}_{f}\right|_{2-k} \gamma\right)(z)\right)=D^{k-1} \mathcal{F}_{f}(z)-\left.\left(D^{k-1} \mathcal{F}_{f}(z)\right)\right|_{k} \gamma,
$$

and our claim is proved. 
Proof of Proposition 3. As it is mentioned in [6, Section 7],the Hecke operators act on harmonic weak Maass forms and on weakly holomorphic modular forms in an analogous way, and, in particular, the formula for the action on Fourier coefficients is the same. If $g=g^{-}+\sum_{n>>-\infty} b(n) q^{n} \in N_{l}$, then $g^{+} \mid T_{m}=\sum_{n>>-\infty} b_{m}(n) q^{n}$ with

$$
b_{m}(n)=\sum_{d \mid(n, m)} d^{l-1} b\left(n m / d^{2}\right) .
$$

It is also easy to verify that

$$
\xi_{l}\left(g \mid T_{m}\right)=m^{1-l}\left(\xi_{l}(g)\right) \mid T_{m} .
$$

It follows from (6) that $\xi\left(g_{m}-m^{k-1} g_{1} \mid T_{m}\right)=0$ for $m>0$, and therefore, by [5, Proposition 3.2], $g_{m}-m^{k-1} g_{1} \mid T_{m} \in M_{2-k}^{!}$. Since $g_{m}-m^{k-1} g_{1} \mid T_{m}$ has no poles neither in the upper half-plane, nor in cusps, and $k>2$, it must be zero:

$$
g_{m}=m^{k-1} g_{1} \mid T_{m}
$$

It now follows from (7) that for positive $m$ and $n$

$$
b(m, n)=m^{k-1} \sum_{d \mid(n, m)} d^{1-k} b\left(1, n m / d^{2}\right) .
$$

Since

$$
g_{m}=g_{m}^{-}+q^{-m}+\sum_{n \geq 0} b(m, n) q^{n} ; \quad D^{k-1} g_{n}^{+}=-n^{k-1} q^{-n}+\sum_{l \geq 1} l^{k-1} b(n, l) q^{l},
$$

we deduce using (9) that

$$
\left\{D^{k-1} g_{n}^{+}, g_{m}\right\}=m^{k-1} b(n, m)-n^{k-1} b(m, n)=0 .
$$

Proof of Corollary 1. The formulas (2), (7) and (8) imply that

$$
f_{n}=-n^{1-k} f_{1} \mid T_{n}
$$

This identity along with (9) reduces the proof to the case $n=1$. The latter case is easy: for example, let

$$
\psi=f_{1}-\left(\frac{E_{k+11}}{\Delta}-t E_{k-1}\right)
$$

where $\Delta=q \prod_{n \geq 1}\left(1-q^{n}\right)^{24}$ is the unique normalized cusp form of weight 12 , and $t$ is the constant term of the $q$-expansion of the ratio $E_{k+11} / \Delta$. Note that the cusp form $\psi$ which satisfies Corollary 1 is not unique.

\section{Acknowledgements}

The author thanks the referee for thoughtful comments. 


\section{References}

[1] T. Asai, M. Kaneko, and H. Ninomiya, Zeros of certain modular functions and an application, Comment. Math. Univ. St. Paul. 46 (1997), no. 1, 93-101.

[2] G. Bol, Invarianten linearer Differentialgleichungen, Abh. Math. Sem. Univ. Hamburg 16 (1949) 1-28.

[3] K. Bringmann and K. Ono, Arithmetic properties of coefficients of half-integral weight MaassPoincaré series, Math. Ann. 337 (2007), no. 3, 591-612.

[4] - Lifting cusp forms to Maass forms with an application to partitions, Proc. Natl. Acad. Sci. USA 104 (2007), no. 10, 3725-3731.

[5] J. H. Bruinier and J. Funke, On two geometric theta lifts, Duke Math. J. 125 (2004), no. 1, 45-90.

[6] J. H. Bruinier and K. Ono, Heegner divisors, L-functions and harmonic weak Maass forms, preprint.

[7] W. Duke and P. Jenkins, On the zeros and coefficients of certain weakly holomorphic modular forms, Pure and Applied Mathematics Quarterly 4 (2008), no. 4, 1327-1340.

[8] J. D. Fay, Fourier coefficients of the resolvent for a Fuchsian group, J. Reine Angew. Math. 293/294 (1977) 143-203.

[9] A. Folsom and K. Ono, Duality involving the mock theta function $f(q)$, J. Lond. Math. Soc. (2) 77 (2008) 320-334

[10] D. A. Hejhal, The Selberg trace formula for $\operatorname{PSL}(2, R)$. Vol. 2, Vol. 1001 of Lecture Notes in Mathematics, Springer-Verlag, Berlin (1983).

[11] D. Niebur, A class of nonanalytic automorphic functions, Nagoya Math. J. 52 (1973) 133-145.

[12] D. Zagier, Traces of singular moduli, in Motives, polylogarithms and Hodge theory, Part I, Vol. 3 of Int. Press Lect. Ser., 211-244, Int. Press, Somerville, MA (2002). Irvine, CA. 1998.

Department of Mathematics, University of Hawait, 2565 McCarthy Mall, Honolulu, HI, 96822-2273

E-mail address: pavel@math.hawaii.edu 